\title{
Recent Advances and Perspectives in Cancer Drug Design
}

\author{
LUMA G. MAGALHAES, LEONARDO L.G. FERREIRA and ADRIANO D. ANDRICOPULO
}

Laboratório de Química Medicinal e Computacional, Centro de Pesquisa e Inovação em Biodiversidade e Fármacos, Instituto de Física de São Carlos, Universidade de São Paulo, Av. João Dagnone, 1100, Jd. Santa Angelina, 13563-120 São Carlos, SP, Brazil

Manuscript received on October 17, 2017; accepted for publication on December 18, 2017

\begin{abstract}
Cancer is one of the leading causes of death worldwide. With the increase in life expectancy, the number of cancer cases has reached unprecedented levels. In this scenario, the pharmaceutical industry has made significant investments in this therapeutic area. Despite these efforts, cancer drug research remains a remarkably challenging field, and therapeutic innovations have not yet achieved expected clinical results. However, the physiopathology of the disease is now better understood, and the discovery of novel molecular targets has refreshed the expectations of developing improved treatments. Several noteworthy advances have been made, among which the development of targeted therapies is the most significant. Monoclonal antibodies and antibody-small molecule conjugates have emerged as a worthwhile approach to improve drug selectivity and reduce adverse effects, which are the main challenges in cancer drug discovery. This review will examine the current panorama of drug research and development (R\&D) with emphasis on some of the major advances brought to clinical trials and to the market in the past five years. Breakthrough discoveries will be highlighted along with the medicinal chemistry strategies used throughout the discovery process. In addition, this review will provide perspectives and updates on the discovery of novel molecular targets as well as drugs with innovative mechanisms of action.
\end{abstract}

Key words: Cancer, targeted therapy, cytotoxic therapy, drug discovery.

\section{INTRODUCTION}

When the nitrogen mustard alkylating agent mechlorethamine reached the market in 1949 as the first anticancer drug, the worldwide life expectancy was 46.8 years (World Population Prospects The 2015 Revision, Kinch 2014). In 2015, when approximately 160 anticancer drugs were available for clinical use, the life expectancy reached 71.4

Correspondence to: Adriano D. Andricopulo

E-mail: aandrico@ifsc.usp.br

* Contribution to the centenary of the Brazilian Academy of Sciences. years (World Health Statistics 2017). However, an increase in the number of deaths associated with aging diseases, such as cancer, resulted from this demographic change (Ames et al. 1993). As a consequence, cancer is the second leading cause of death worldwide today (World Health Statistics 2017). Therefore, the constant development of novel antineoplastic agents continues to be a foremost public health demand, despite the current availability of many anticancer drugs in the market.

The history of rational drug design can have its roots traced back to the awarding of the Nobel 
Prize to George H. Hitchings and Gertrude Elion after their contribution in understanding nucleic acid metabolism in normal and neoplastic human cells (Baguley and Kerr 2001). They revolutionized drug discovery by bringing to the field principles of biochemistry and physiology, which have helped in understanding the molecular basis of several diseases. For example, the structure of purines required for synthesis of DNA offered the basis for developing the chemotherapeutic agent 6-mercaptopurine (Raju 2000). At the same time, folic acid antagonists developed by Sidney Farber, such as 4-amino-pteroylglutamic acid, entered the clinic for treating child leukemia (Miller 2006). These landmark developments prompted a new paradigm in cancer therapy marked by the use of molecular approaches to disclose the cellular mechanisms involved in neoplastic transformation.

Since then, the number of anticancer agents reaching the market has gradually increased. On average, two new oncologic drugs per year have hit the market between 1950 and 1980 (Kinch 2014). This number doubled in the 1990s and has constantly increased since then, reaching an average of 10 new drugs per year between 2011 and 2016 (Kinch 2014). These numbers can be explained by the fact that pharmaceutical companies have unprecedented investments in cancer research as a result of the constant growth in the worldwide demand for antineoplastic agents. This rising demand led to a remarkable increase in the number of sales in this therapeutic area. For instance, paclitaxel, a highly prescribed microtubule stabilizer, was the first anticancer drug to reach annual sales as high as $\$ 1$ billion in the late 1990s (Goodman and Walsh 2001). Currently, the top 10 best-selling antineoplastic drugs have registered annual sales ranging from $\$ 1.8$ billion to almost $\$ 8$ billion (Prasad et al. 2017).

Not only have the sales of drugs notably increased but also the treatment costs; treating one single patient can cost approximately $\$ 10,000$ per month, which is a significant increase compared to the $\$ 100$ per month in 1960 (Prasad et al. 2017, Bach 2009). This relevant growth has come alongside the sharply rising costs of drug R\&D (research and development), which is usually associated with a steep increase in the complexity of the R\&D process observed in the past two decades. In fact, this steep increase in $R \& D$ cost can be reasoned as a downside of the industry's focus on highly complex diseases and the ever-increasing dependence of the sector on cutting-edge knowledge and innovative and hugely expensive technologies. The complexity of the newly launched drugs can be attributed to another reason. Many of these agents are biological molecules, e.g., monoclonal antibodies (mAbs) or antibody-drug conjugates (ADCs), which are much more expensive to produce than small-molecule drugs.

This increasing drug demand resulted in an enormous diversification of the drug discovery approaches in all therapeutic areas, particularly cancer. Over the last 60 years, different strategies relying on very different biological and molecular aspects of neoplastic transformation have been used in pharmaceutical R\&D. This review will provide an overview of the mechanisms of action/molecular targets of anticancer compounds. Moreover, recent and successful discovery cases will be examined in detail, providing an up-to-date perspective on cancer drug research.

\section{CYTOTOXIC AND TARGETED CANCER THERAPY}

Compounds acting as antimetabolites, DNAalkylating and microtubule-interfering agents and those blocking DNA synthesis are classified as cytotoxic drugs (Dumontet and Jordan 2010, Magalhaes et al. 2016). These molecules work as poorly selective poisons and, despite their effectiveness, present a narrow therapeutic window (Chari 2007). The pioneering antitumor drugs, which were developed in the late 1940s, belong to 
this class. Another type of oncologic drug is called targeted therapy, which consists of molecules that modulate the activity of proteins specifically involved in tumorigenesis and cancer progression (Aggarwal 2010, Joo et al. 2013). Examples of these drugs are mAbs and small molecules that interfere with specific pathways associated with malignant transformation. In general, mAbs do not penetrate the cellular membrane, but instead, they act on proteins located on the cell surface. Alternatively, small molecules penetrate the cell and interfere with signaling pathways by acting on intracellular molecular targets (Joo et al. 2013). A notable small-molecule targeted therapy is imatinib, which is a selective inhibitor of BRC-Abl tyrosine kinase that is overexpressed in chronic myeloid leukemia (CML) (Deininger et al. 2005). Imatinib is a pioneering targeted therapy and a breakthrough in cancer drug discovery. Imatinib has achieved enormous success in clinical practice, being approved and widely prescribed for more than eight cancer types (Prasad et al. 2017, Aggarwal 2010).

Encouraged by the success of imatinib, several biologics and small molecules have been developed as new anticancer agents, such as sunitinib, erlotinib and rituximab (Aggarwal 2010). These novel agents represented important developments in cancer treatment, however, some of them, such as bevacizumab, had disappointing clinical results when used as monotherapies (Aggarwal 2010, Deininger et al. 2005). This lack of effectiveness can be enlightened by some wellknown limitations of targeted therapeutic agents. The main drawbacks consist of the development of resistance mechanisms, such as mutations in the molecular target, activation of alternative biochemical pathways, and the low cytotoxic power of these compounds (Aggarwal 2010). Once these limitations have been better understood, most of the targeted agents are used in combination with another targeted drug or cytotoxic compound. This strategy mitigates the shortcomings associated with both drugs and potentiates the benefits of each one, ultimately improving the clinical outcome (Masui et al. 2013). One successful example is the combined use of bevacizumab, oxaliplatin and 5-fluorouracil to treat metastatic colorectal cancer (Aggarwal 2010). Another example is the combined use of trastuzumab and docetaxel as a first-line treatment for anti-human epidermal growth factor receptor 2 (HER2)-positive breast cancer (Redana et al. 2010).

The use of antibodies as a system for selectively delivering cytotoxic drugs to tumor cells is another approach that explores the synergy between targeted and cytotoxic compounds. ADC therapy aims to reduce the side effects associated with the non-selective nature of cytotoxic drugs (Chari 2007). The conjugate is designed to remain nontoxic until it reaches the target neoplastic tissue. To achieve this goal, the ADC is composed of several components: (i) an antibody that confers high specificity for antigens overexpressed specifically by tumor cells; (ii) a linker that maintains the stability of the system until the ADC reaches the target cell; (iii) and a drug that needs to be water soluble, potent and amenable to be attached to the linker. This system allows the selective delivery of the drug to cancer cells upon the cleavage of the linker (Chari 2007). Thus, the development of effective ADCs requires the optimization of these three components. Two FDA-approved ADCs are currently available: brentuximab-vedotin (approved in 2011) and ado-trastuzumab emtansine (T-DM1; approved in 2013).

\section{MECHANISM OF ACTION OF FDA- APPROVED ANTICANCER DRUGS}

To understand the evolution of pharmaceutical cancer research, all of the 166 FDA-approved oncologic drugs, excluding vaccines and adjuvants, were categorized based on their mechanism of action (Fig. 1a). Accordingly, these drugs were 
divided into 11 categories based on their molecular targets. Each drug was allocated into only one category, even those acting on multiple targets, taking into account the main mechanism of action. Small molecules, mAbs, proteins and ADCs cover $80 \%, 16 \%, 3 \%$, and $1 \%$ of the total number of drugs, respectively. Compounds interacting with kinases represent $28 \%$ of this total and consist of the major category. DNA-alkylating/intercalating agents, immunotherapies and antimetabolites represent $13 \%, 11 \%$, and $10 \%$ of this therapeutic space, respectively. DNA-interfering agents and antimetabolites are the pioneering classes of anticancer drugs, but this is not translated into a quantitative predominance among the universe of anticancer drugs. However, kinase-interacting drugs, which started to hit the market in the 2000s following the success of imatinib and the optimism about targeted therapy, represent the predominant therapeutic mechanism. Most of the targeted kinases are tyrosine kinases (75\%) and HER2, epidermal growth factor receptors (EGFR), vascular endothelial growth factor receptors (VEGFR) and platelet-derived growth factor receptors (PDGFR) (42\%). Vascular-disrupting/anti-angiogenic agents represent an important phenotypic mechanism of action. A total of $15 \%$ of the anticancer drugs acts by this mechanism, which is associated with the modulation of different molecular targets, such as VEGFR and tubulin (Tozer et al. 2005).

The use of mAbs is a relatively recent phenomenon that can be traced back to 1997 when rituximab broke into the market as the first $\mathrm{mAb}$ approved for use in oncologic patients (Oldham and Dillman 2008). Today, mAbs represent $16 \%$ of the available therapeutic options. Generally, mAbs are categorized according to their mechanism of action (Fig. 1b): (i) immunotherapeutics (45\%), which trigger the immune system to eliminate cancer cells by a diversity of mechanisms, for example, antibody-dependent cellular cytotoxicity (ADCC) (Petrelli and Giordano 2008); and (ii)
mAbs $(24 \%)$, which produce their antitumoral effects by interacting with growth factor receptor kinases.

\section{CURRENT PANORAMA OF CANCER DRUG RESEARCH (2013 - 2017)}

To evaluate the recent evolution of pharmaceutical R\&D for cancer, drug approvals from 2013 to 2017 were highlighted (Fig. 1c). A total of 33 new drugs have entered the market during this period, among which $61 \%$ are small molecules, $36 \%$ consist of mAbs and 3\% are ADCs. These numbers show that $45 \%$ of all mAbs and ADCs were approved in the past five years, which demonstrates the current interest of the industry in this field. Kinases remain as the main molecular target, covering $37 \%$ of the latest approvals. Among the kinases, 25\% are growth factor receptors, which comprise $9 \%$ of the total number of new drugs.

The high number of mAb approvals demonstrates the current importance of immunotherapy, which is the second major therapeutic class, representing $30 \%$ of the last fiveyear approvals. Among mAbs, including ADCs, $77 \%$ are immunotherapeutics, $15 \%$ consist of growth factor kinase inhibitors, and $8 \%$ are tubulin inhibitors (Fig. 1d). Immunotherapeutics include pembrolizumab and nivolumab, which were launched in 2014 and are the earliest drugs to act as inhibitors of the immune checkpoint programmed cell death protein 1 (PD1). Moreover, atezolizumab, avelumab and durvalumab (launched 2016 2017) are the pioneer inhibitors of the immune checkpoint programmed death ligand 1 (PDL1). Vascular-disrupting/anti-angiogenic agents remain an important class, consisting of $21 \%$ of the new drugs.

Ado-trastuzumab emtansine, an ADC approved within the last five years, is conjugated with a cytotoxic compound (maytansinoid DM1) that targets tubulin, which is the same mechanism 

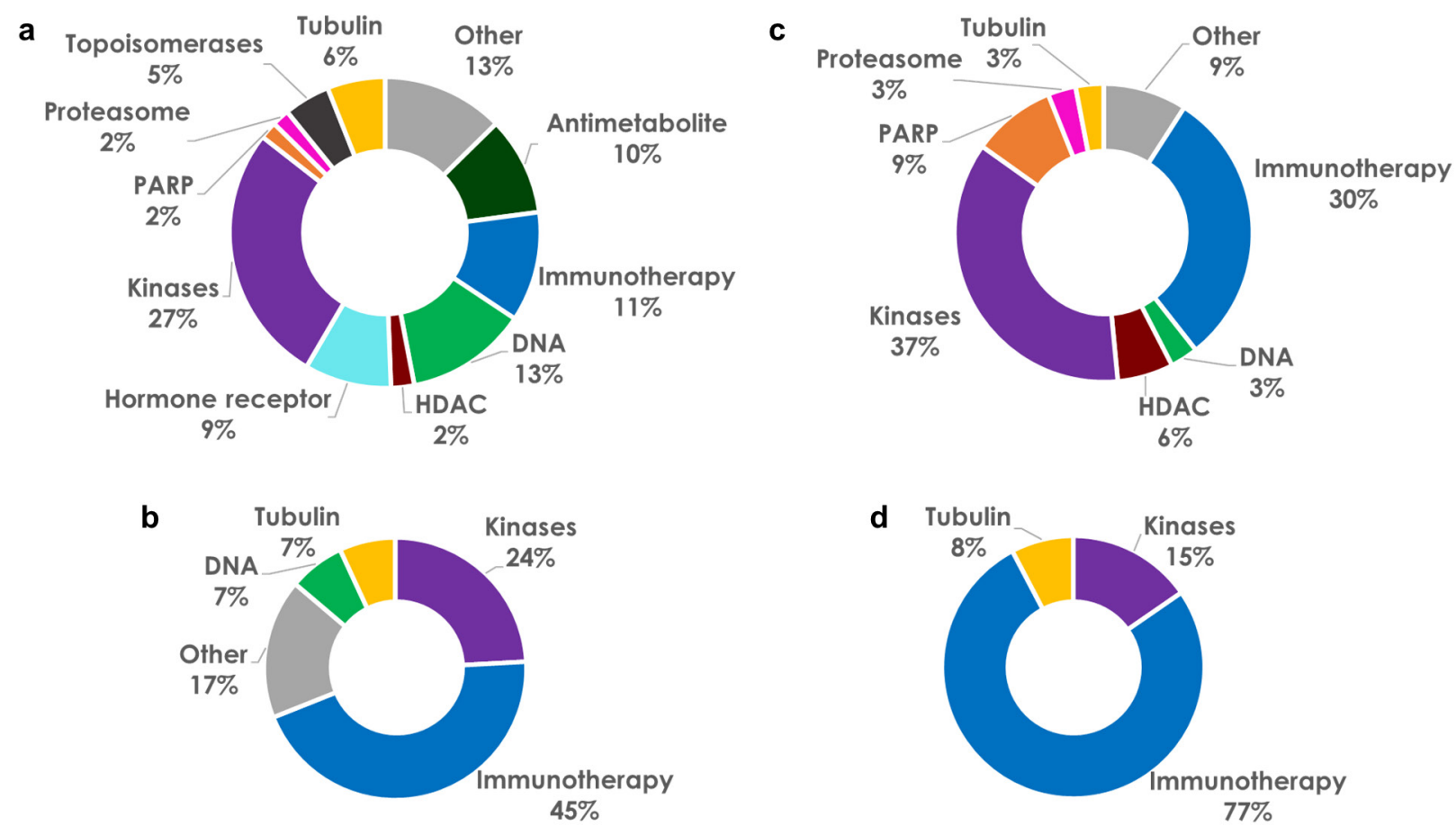

Figure 1 - Mechanistic analysis of anticancer drugs. (a) Mechanistic basis for all FDA-approved cancer drugs. (b) Mechanistic classification of all FDA-approved mAbs. (c) Mechanistic basis for FDA-approved anticancer drugs in the past five years. (d) Mechanistic classification of FDA-approved mAbs in the past five years. Abbreviations - PARP: poly (ADP-ribose) polymerase, HDAC: histone deacetylases.

of the cytotoxic agent monomethyl auristatin E conjugated to brentuximab-vedotin. Another class of drugs that debuted in this period consists of the following inhibitors of poly(ADP-ribose) polymerases (PARPs), which are enzymes involved in DNA repair (Beck et al. 2017): olaparib, rucaparib and niraparib (approved in 2014, 2016, and 2017, respectively). Another notable recent development is venetoclax (2016), the first inhibitor of B cell lymphoma 2 (Bcl-2) proteins, which are key regulators of programmed cell death (apoptosis). By inhibiting Bcl-2 proteins, venetoclax prevents cancer cells from undergoing uncontrolled reproduction (Scott et al. 2016).

An overall analysis of the mechanism of action of drugs that recently entered the clinic highlights targeted therapy as the main focus of current cancer R\&D. Except for the radiopharmaceutical radium RA 223 dichloride (approved in 2013) and the
DNA-alkylating agent trabectedin (approved in 2015), all recently developed drugs act as targeted therapeutics.

\section{CANCER DRUG DISCOVERY APPROACHES}

Drug discovery is a remarkably challenging field, and developing novel treatments for cancer has its specific hurdles that are associated with the complexity of the disease. Among the main obstacles is the development of molecules that selectively interact with the neoplastic tissue. Several recent reports have stated that, despite the development of novel drugs with novel mechanisms of action, the benefits for the patient are still modest in general. The average gain in progression-free and overall survival, which are commonly used indicators to evaluate the clinical benefit of anticancer therapies, are as low as 2.5 and 2.1 months, respectively 
(Prasad et al. 2017, Toniatti et al. 2014, Fojo et al. 2014, Sullivan and Aggarwal 2016).

The delivery of a new drug takes approximately 11 years (DiMasi et al. 2016) and can be summarized according to the following workflow. The preclinical phase involves the following: (i) selection and validation of the molecular or phenotypic target; (ii) identification of a hit molecule and hit-to-lead optimization; and (iii) optimization of the lead to a clinical candidate. Next, the candidate is evaluated for safety, pharmacokinetics and efficacy in humans in the clinical development phase.

In the hit identification step, experimental and computational techniques can be used, such as high-throughput screening (HTS) and virtual screening (VS), respectively (Moffat et al. 2014, Bhinder and Djaballah 2014). Once a hit is identified, it is optimized to a lead compound and ultimately to a clinical candidate. This is carried out by subjecting the compound to a process that involves the outlining of molecular modifications, synthesis of analogs and an extensive computational and experimental profiling, which are ultimately used to establish structure-activity relationships (SAR) among the designed series. This process occurs to optimize both pharmacodynamics (PD) and pharmacokinetics (PK) properties, which are key requirements for generating well-characterized and suitable clinical candidates (Colombo and Peretto 2008). Diverse strategies are applied in molecular design and optimization. These methods are divided into structure- and ligand-based drug design (SBDD and LBDD, respectively). SBDD approaches, such as X-ray crystallography, molecular docking, and molecular dynamics are used to disclose the intermolecular interactions that occur in the formation of the ligand-receptor complex. LBDD strategies, such pharmacophore, quantitative structure-property relationship (QSPR) and quantitative structure-activity relationship (QSAR) models are widely used to identify and predict relevant ligand properties for PD and PK
(Merz et al. 2010). Optimized compounds are then evaluated in preclinical animal models of the disease to access PK, toxicity and efficacy parameters. In cancer research, selecting a suitable in vivo model is a critical task. An inadequate model frequently results in misleading data and false-positive candidates that fail in the clinical development phase (Talmadge et al. 2007). Ideally, at least one orthotropic or xenograft model must be used, followed by the evaluation in the autochthonous or genetically engineered in vivo model (GEM) (Talmadge et al. 2007, Richmond and $\mathrm{Su} 2008$ ). Once the gathered preclinical data qualify the compound as a clinical candidate, it can move to the clinical development phase, in which its efficacy and safety will be evaluated in human cohorts; which requires the application of an Investigational New Drug (IND).

\section{RECENT TRENDS IN CANCER RESEARCH}

Recent case studies in cancer research will be highlighted in the following sections. Additionally, the medicinal chemistry strategies employed to develop compounds that interact with relevant molecular targets, the discovery process of certain approved drugs, and the methods used to approach challenging targets will be discussed. Drugs approved in the past five years will be the focus of the selected case studies. All of the examples that will be examined are targeted therapies that have a breakthrough mechanism of action. The selected case studies represent recent advances in cancer drug $R \& D$ that have been achieved by the use of distinct medicinal chemistry and drug discovery approaches. The criteria for electing the molecular targets that will be discussed relied on: (i) novelty of the molecular target (breakthrough therapies); (ii) availability of compound optimization data; and (iii) coverage of different drug R\&D strategies. 


\section{Selective kinase Cdk4/6 inhibitors}

Cyclin-dependent kinases (Cdks), which are enzymes that control the cell cycle, emerged as potential molecular targets for cancer therapy approximately 20 years ago. Among the Cdk family, $\mathrm{Cdk} 7, \mathrm{Cdk} 8$ and $\mathrm{Cdk} 9$ regulate transcription, while Cdk1, Cdk2, Cdk4 and Cdk6 promote cell cycle progression (Asghar et al. 2015). Cdk4 and Cdk6 regulate the cell cycle checkpoint that drives the progression from the $G_{1}$ or $G_{0}$ phases into the $S$ phase, in which DNA replication begins (Asghar et al. 2015, Chen et al. 2016). After an extracellular signal initiates the transition to $\mathrm{S}$ phase, Cdk4/6 proteins phosphorylate key substrates, such as tumor suppressor retinoblastoma protein (RB). These substrates bind to E2F transcription factors, thereby regulating the expression of several genes involved in cell cycle control (e.g., cyclins A and E) (Chen et al. 2016). The Cdk4/6-RB pathway is often disrupted in cancer, which results in uncontrolled cell division. Therefore, Cdk4/6 proteins have been actively explored in cancer drug discovery efforts (Tripathy et al. 2017).

The first generation of molecules targeting the Cdk family are pan-Cdk inhibitors, such as flavopiridol and roscovitine, which failed in clinical trials in the 1990s (Chen et al. 2016). Second generation inhibitors, such as dinaciclib, are more potent and slightly selective against Cdk1 and Cdk2. However, toxicity remains a critical drawback of these drugs because many Cdks are essential for the survival of healthy cells (e.g., Cdk1 and Cdk9) (Asghar et al. 2015). Third generation Cdk inhibitors, such as palbociclib (approved in 2015) and ribociclib (approved in 2017) are selective $\mathrm{Cdk} 4 / 6$ inhibitors. The development of palbociclib started with the identification of pyridopyrimidinones (Fig. 2a), which are moderately selective Cdk inhibitors (Barvian et al. 2000). Kinetic assays demonstrated that these molecules are competitive inhibitors that interact with the ATP binding site in Cdks.

To achieve selectivity, novel pyridopyrimidinones (compounds $\mathbf{1}$ and 2, Fig. 2a) were designed and evaluated in enzyme kinetic assays using Cdk2 and Cdk4 (Toogood et al. 2005). The first selective Cdk4 inhibitor was obtained by replacing the aniline group of compound $\mathbf{3}$ (Fig. 2a) with an aminopyridine (compound 4) at the $\mathrm{C} 2$ position. This change in the selectivity profile also occurred between compounds $\mathbf{5}$ and 6 (Toogood et al. 2005). Next, SAR studies were conducted by introducing diverse modifications in the structure of the reference molecule (compound 6). A cyclopentyl group was identified as the most suitable substituent at N8 (compound 7). An equilibrium between reasonable enzyme inhibition and aqueous solubility was achieved by adding a methyl group at C5 and an acetyl group at C6, resulting in compound $\mathbf{8}$ (palbociclib). Moreover, this compound had a half maximal inhibitory concentration $\left(\mathrm{IC}_{50}\right.$ ) value of $160 \mathrm{nM}$ against breast cancer MDA-MB-435 cells, causing negligible cytotoxicity in the RB-negative MDA-MB-468 cell line (Fig. 2a) (Toogood et al. 2005).

Further studies showed that compound $\mathbf{8}$ was the only Cdk inhibitor among the designed series that had the ability to arrest cancer cells exclusively at the $\mathrm{G}_{1}$ phase (Toogood et al. 2005). Additionally, inhibition studies showed remarkable selectivity for Cdk4/6 $\left(\mathrm{IC}_{50}=10 \mathrm{nM}\right)$ over 36 different kinases $\left(\mathrm{IC}_{50}>10 \mu \mathrm{M}\right)(\mathrm{Lu}$ and Schulze-Gahmen 2006). After characterization against different breast cancer cell lines, compound $\mathbf{8}$ was progressed to clinical trials to be evaluated for its ability to treat ER-positive luminal breast cancer and HER2amplified disease (Finn et al. 2009).

Ribociclib, another selective inhibitor of Cdk4/6 that is structurally similar to palbociclib (Fig. 2c), was approved in 2017. X-ray studies on this class of compounds in complex with Cdks provided the structural data required for 
<smiles>[R]n1c(=O)ccc2cnc(Nc3ccc(N4CCN(C)CC4)cc3)nc21</smiles><smiles>O=c1c(Br)cc2cnc(Nc3ccc(N4CCNCC4)cc3)nc2n(C2CCCC2)c1=O</smiles>

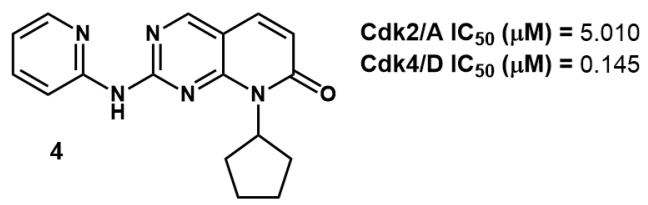<smiles>O=c1c(Br)cc2cnc(Nc3ccc(N4CCNCC4)cn3)nc2n1C1CCCC1</smiles>

Cdk2/E IC $50(\mu \mathrm{M})>5$

$\mathrm{Cdk}_{4} / \mathrm{D}$ IC $50(\mu \mathrm{M})=0.016$

FGFr $\quad \mathrm{IC}_{50}(\mu \mathrm{M})>5$

PDGFr IC $50(\mu \mathrm{M})>5$<smiles>O=c1ccc2cnc(Nc3ccc(N4CCNCC4)cn3)nc2n1C1CCCC1</smiles>

Cdk2/A IC $50(\mu \mathrm{M})=2.5$ $\mathrm{Cdk}_{4} / \mathrm{D}$ IC $\mathrm{C}_{50}(\mu \mathrm{M})=0.015$<smiles>CC(=O)c1c(C)c2cnc(Nc3ccc(N4CCNCC4)cn3)nc2n(C2CCCC2)c1=O</smiles>

Cdk2/A $\quad \mathrm{IC}_{50}(\mu \mathrm{M})>5$

Cdk4/D $\quad I C_{50}(\mu \mathrm{M})=0.011$

MDA-MB-435 IC $50(\mu M)=0.160$

MDA-MB-468 $\quad \mathrm{IC}_{50}(\mu \mathrm{M})>3$
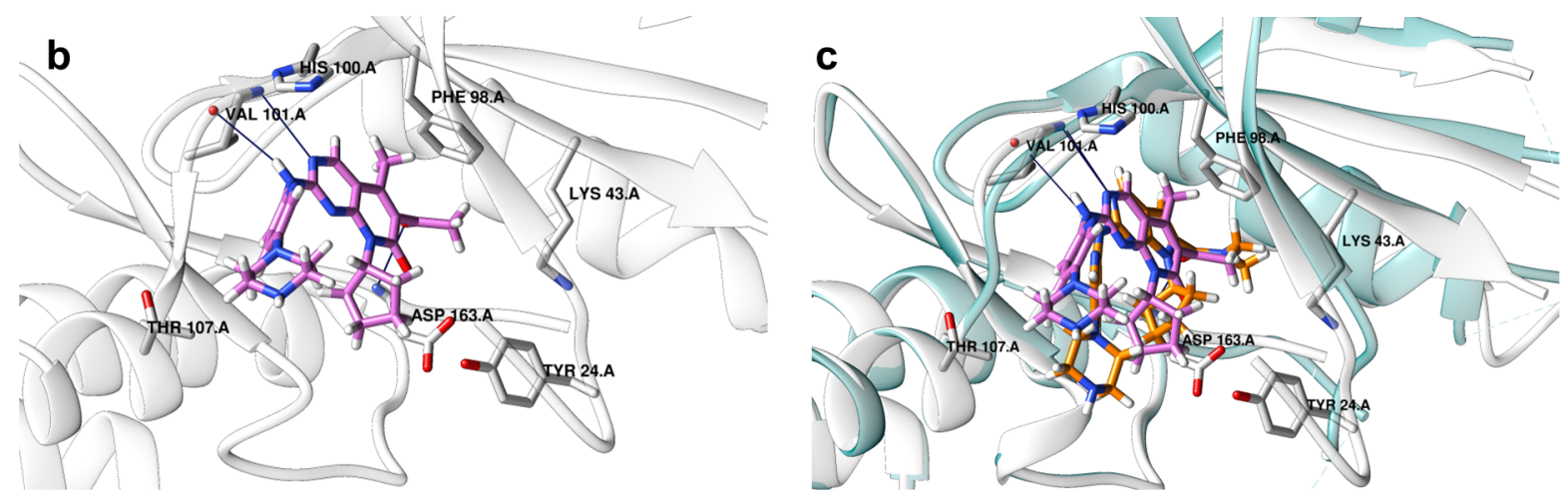

Figure 2 - Cdk4/6 inhibitors. (a) Relevant compounds synthesized during palbociclib development. (b) Cdk6 cocrystal structure for palbociclib (PDB 5L2I). Key residues are labeled. (c) Superposition of Cdk6 cocrystal structures for palbociclib (PDB 5L2I) and ribociclib (PDB 5L2T) showing the similarity of binding mode and intermolecular interactions with relevant residues for selectivity towards Cdk6. Abbreviations - Cdk: cyclin-dependent kinase, FGFr: fibroblast growth factor receptor, PDGFr: plateletderived growth factor receptor, $\mathrm{IC}_{50}$ : half maximal inhibitory concentration. 
the development of selective Cdk4/6 inhibitors. Key structural aspects were observed, such as the interaction of these inhibitors with the residues His 100 and Thr107 near the hinge region in Cdks (Fig. 2b and c). Another important feature considered in the design process was to avoid the interaction with highly conserved residues across the Cdk family (Chen et al. 2016). The knowledge gathered in the R\&D process of these compounds has provided a solid foundation for the development of new clinically useful Cdk inhibitors.

\section{Antibody-drug conjugates for breast cancer}

The development of effective ADCs requires the design and optimization of three elements: the antibody, the cytotoxic agent and the linker. T-DM1 was designed to treat HER2-positive breast cancer by using trastuzumab, an anti-HER2 antibody (Lambert and Chari 2014). Trastuzumab was approved for use in HER2-positive breast cancer therapy in 1998 and is often administered in combination with paclitaxel or docetaxel, two microtubule stabilizers. When combined with these drugs, trastuzumab is effective in eliminating cancer cells by an antibody-dependent cytotoxicity mechanism; however, some tumors do not respond satisfactorily to the treatment, and most patients who are responsive at the beginning of therapy still experience disease progression (Nahta and Esteva 2003). In view of these findings, T-DM1 was developed with the aim of improving the cytotoxicity of trastuzumab while taking advantage of its tumor selectivity.

The selection of the cytotoxic agent to be attached to trastuzumab was initially guided by the success of the combination therapy trastuzumabpaclitaxel/docetaxel. Conjugation with other tubulin modulators, e.g., vinblastine, was the first attempt, which resulted in disappointing results (Lambert and Chari 2014). The conjugation process usually resulted in ADCs that were 8-fold less potent than the parental free drug. These findings led to the search for compounds featuring higher cytotoxicity (half-maximal cytotoxic concentration, $\mathrm{CC}_{50} \approx$ $0.01 \mathrm{nM}$ ), which resulted in the identification of maytansine (9), a benzoansamacrolide isolated from Maytenus ovatus that is a remarkably potent inhibitor of tubulin polymerization ( $\mathrm{IC}_{50}$ of $30-$ $100 \mathrm{pM}$ for a panel of tumor cell lines) (Jordan et al. 1998). Additionally, maytansine exhibits adequate aqueous solubility and stability, which are key factors for developing clinically useful ADCs.

Functionalization of the cytotoxic agent is required to enable the conjugation process. A thiol group was selected because of its amenability to participate in commonly used coupling reactions (Lambert and Chari 2014). Next, a series of C3 disulfide-containing maytansine derivatives was synthesized, and the cytotoxicity of these derivatives on human cancer cell lines was assessed. All of the derivatives had similar or higher potency than maytansine (compounds 10 - 12, Fig. 3a) (Chari et al. 1992).

The linker is another critical element of an $\mathrm{ADC}$ and must be stable in the bloodstream and efficiently cleaved inside the target cells (Fishkin et al. 2011). Disulfide and thioether linkers (compounds 13 - 16, Fig. 3b) were proposed for conjugation with lysine residues in the antibody (Lewis Phillips et al. 2008). The ratio of linked maytansinoids per antibody was optimized (3.5 molecules per antibody) to minimize the amount of unconjugated antibody and to avoid excessive maytansinoid loading, which could result in poor solubility (Lewis Phillips et al. 2008).

The cytotoxicity profile of five conjugates (compounds 17 - 21, Fig. 3c) was assessed using HER2-amplified cell lines (BT-474 and SK-BR-3). Similar activities for all linkers and an improvement in potency (more than 100-fold compared with the unconjugated antibody) were observed (Lewis Phillips et al. 2008). Cells that do not express HER2 (MDA-MB-486) were more than 200-fold 

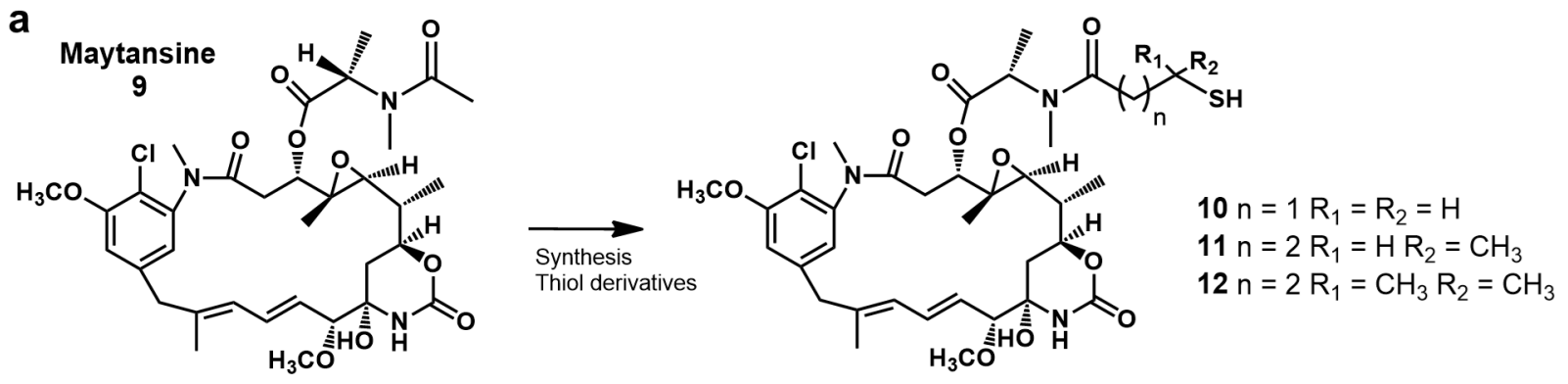

SK-Br-3 cells $I C_{50}(n M)=0.030$

b<smiles>O=C(CCSSc1ccccn1)ON1C(=O)CCC1=O</smiles>

C

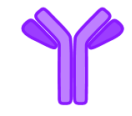

Trastuzumab<smiles>CC(CCC(=O)ON1C(=O)CCC1=O)SSc1ccccn1</smiles>

14<smiles>CC(CCC(=O)ON1C(=O)C[C@H](S(=O)(=O)O[Na])C1=O)SSc1ccc([N+](=O)[O-])cn1</smiles>

15

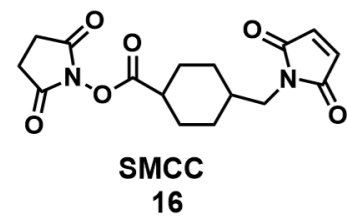

SMCC 
The linkers SMCC and SPP yielded similar results and were therefore selected for tolerability studies. The SMCC-containing ADC was better tolerated, and considering the overall preclinical data, it was selected as the clinical candidate (Lambert and Chari 2014). After the clinical trials, T-DM1 was approved in 2013 for HER2-positive breast cancer treatment.

\section{Poly(ADP-ribose) polymerase (PARP) inhibitors}

Cells have a sophisticated machinery for DNA repair. Several mechanisms are available for fixing DNA single-strand and double-strand breaks (SSB and DSB, respectively), such as base excision repair (BER) and homologous recombination (HR). These mechanisms protect cells from endogenousor exogenous-induced DNA errors (Sancar et al. 2004). In addition, DNA repair pathways fix DNA damage caused by chemotherapeutics, which lead to resistance to these agents. Therefore, targeting DNA repair mechanisms has been successfully explored in cancer drug discovery (Jones et al. 2015).

Poly(ADP-ribose) polymerases (PARPs) add poly-ADP-ribose chains (PAR) onto many proteins after DNA single-strand breaks. Addition of these chains relaxes chromatin and signals for the recruitment of DNA repair factors. After PARP dissociation, DNA is repaired through the BER mechanism (Murai et al. 2012). Following these findings, PARPs have been studied as molecular targets for cancer, and the pioneer inhibitors in this class entered clinical trials in the 1990 s as cytotoxic or ionizing radiation sensitizers. The clinical tests were discontinued because of toxicity issues (Jones et al. 2015).

BRCA1 and BRCA2 are genes that encode tumor suppressor proteins involved in the repair of double-strand DNA breaks. Mutations in these genes are a common finding in several types of malignancies, such as breast, ovarian, prostate and pancreatic cancers (Venkitaraman 2002). Since 2005, PARP inhibitors have been demonstrated to induce the death of cells having deficiencies in the BRCA pathway without affecting normal tissue (Jones et al. 2015). In line with these findings, inhibitors of PARP have been studied for treating BRCA1/2-deficient tumors. The development of the earliest PARP inhibitors in the 1990s provided valuable information about the pharmacophore requirements for achieving suitable inhibition profiles towards these enzymes. This is demonstrated by the development of the drugs olaparib, rucaparib and niraparib, which relied on the structure of nicotinamide (compound 22), a weak PARP inhibitor.

Using SBDD strategies, the activity of the newly designed inhibitors was improved by adding rotational restrictions to the molecules, thereby keeping essential interactions with the $\mathrm{NAD}^{+}$ binding site (Fig. 4a) (Wahlberg et al. 2012). For instance, the development of niraparib relied on the restriction of the rotation in the nicotinamide amide group. This effect was achieved by adding a nitrogen atom on the aromatic system, thus inducing the formation of an internal hydrogen bond with the carboxamide group (Jones et al. 2009). To this end, four heteroaromatic carboxamide derivatives were synthesized and evaluated for PARP inhibition. The most active derivative was compound $\mathbf{2 3}$, which displayed an $\mathrm{IC}_{50}$ value of $24 \mathrm{nM}$ in the enzyme inhibition assay and a half maximal effective concentration $\left(\mathrm{EC}_{50}\right)$ of $3700 \mathrm{nM}$ for inhibiting PARylation in a cell-based assay. Relying on these results, new analogs were designed by varying the substituents on the phenyl group (Jones et al. 2009). The best inhibitors (compounds 24 and 25) showed a 7-fold improvement in the enzymatic assay and a 30-fold improvement in the cell-based PARylation test. Better physicochemical properties of compounds 24 and 25, compared with those of the parent compound $\mathbf{2 3}$, and the consequent improved cell penetration explain the better results 


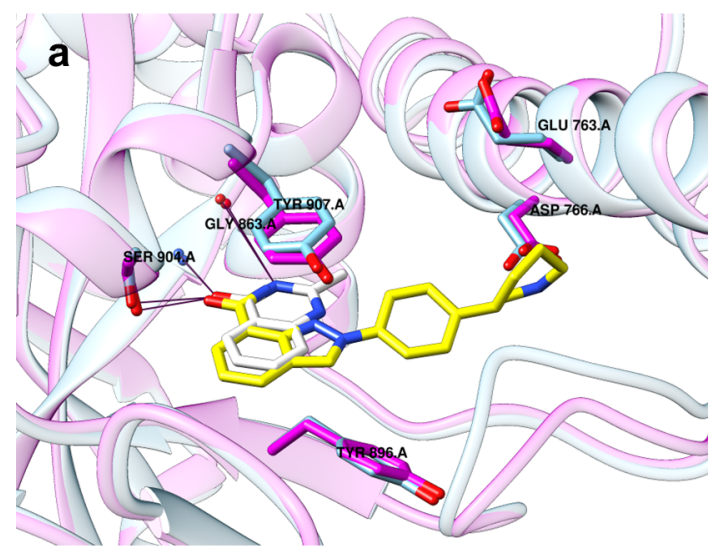<smiles>N#Cc1cccc(C(N)=O)c1</smiles><smiles>NC(=O)c1cccc2cn(-c3ccccc3)nc12</smiles>

23<smiles>CCc1ccc(-n2cc3cccc(C(N)=O)c3n2)cc1</smiles>

24

PARP $\quad \mathrm{IC}_{50}(\mathrm{nM})=3.7$

PARylation $\mathrm{EC}_{50}(\mathrm{nM})=110$

$\mathrm{CL}_{\text {int }}(\mu \mathrm{L} / \mathrm{min} / \mathrm{mg})=177$

$\mathrm{CL}_{\text {rat }}(\mathrm{mL} / \mathrm{min} / \mathrm{kg})=450$

PARP $\quad \mathrm{IC}_{50}(\mathrm{nM})=24$

PARylation $\mathrm{EC}_{50}(\mathrm{nM})=3700$<smiles>CNCc1ccc(-n2cc3cccc(C(N)=O)c3n2)cc1</smiles>

25<smiles>NC(=O)c1cccc2cn(-c3ccc(C4CCCNC4)nc3)cc12</smiles><smiles>NC(=O)c1cccc2cn(-c3ccc([C@H]4CCCNC4)cc3)nc12</smiles>

PARP $\quad \mathrm{IC}_{50}(\mathrm{nM})=2.4$ PARylation $\mathrm{EC}_{50}(\mathrm{nM})=30$ $\mathrm{CL}_{\text {int }}(\mu \mathrm{L} / \mathrm{min} / \mathrm{mg})=7$ $\mathrm{CL}_{\text {rat }}(\mathrm{mL} / \mathrm{min} / \mathrm{kg})=24$

26
PARP $\quad \mathrm{IC}_{50}(\mathrm{nM})=3.8$
PARylation $\mathrm{EC}_{50}(\mathrm{nM})=68$
$\mathrm{CL}_{\text {int }}(\mu \mathrm{L} / \mathrm{min} / \mathrm{mg})=28$
PARP
$\mathrm{IC}_{50}(\mathrm{nM})=3.2$
PARylation $\mathrm{EC}_{50}(\mathrm{nM})=24$
$\mathrm{CL}_{\text {int }}(\mu \mathrm{L} / \mathrm{min} / \mathrm{mg})=11$
$\mathrm{CL}_{\text {rat }}(\mathrm{mL} / \mathrm{min} / \mathrm{kg})=220$
$\mathrm{CL}_{\text {rat }}(\mathrm{mL} / \mathrm{min} / \mathrm{kg})=47$<smiles>CCCc1n[nH]c(=O)c2ccccc12</smiles><smiles>[R2]c1ccc(Cc2n[nH]c(=O)c3ccccc23)cc1C(=O)N1CCCN([R1])CC1</smiles>

$\begin{array}{ll} & \text { PARP-1 IC }_{50}(\mu \mathrm{M}) \\ \mathbf{2 8} \mathrm{R}_{1}=\text { 2-hydroxyethyl } \mathrm{R}_{2}=\mathrm{H} & 0.018 \\ \mathbf{2 9} \mathrm{R}_{1}=\text { 2-hydroxyethyl } \mathrm{R}_{2}=\mathrm{F} & 0.007 \\ \mathbf{3 0} \mathrm{R}_{1}=\text { benzyl } \mathrm{R}_{2}=\mathrm{H} & 0.018 \\ \mathbf{3 1} \mathrm{R}_{1}=H \mathrm{R}_{2}=\mathrm{F} & 0.007 \\ \mathbf{3 2} \mathrm{R}_{1}=\text { ethylsulphonyl } \mathrm{R}_{2}=\mathrm{H} & 0.23\end{array}$

$\mathrm{PF}_{50}$ cellular assay

5.4

18.0

2.2

12.6

1.4

PARP IC $_{50}(\mu \mathrm{M})=0.77$
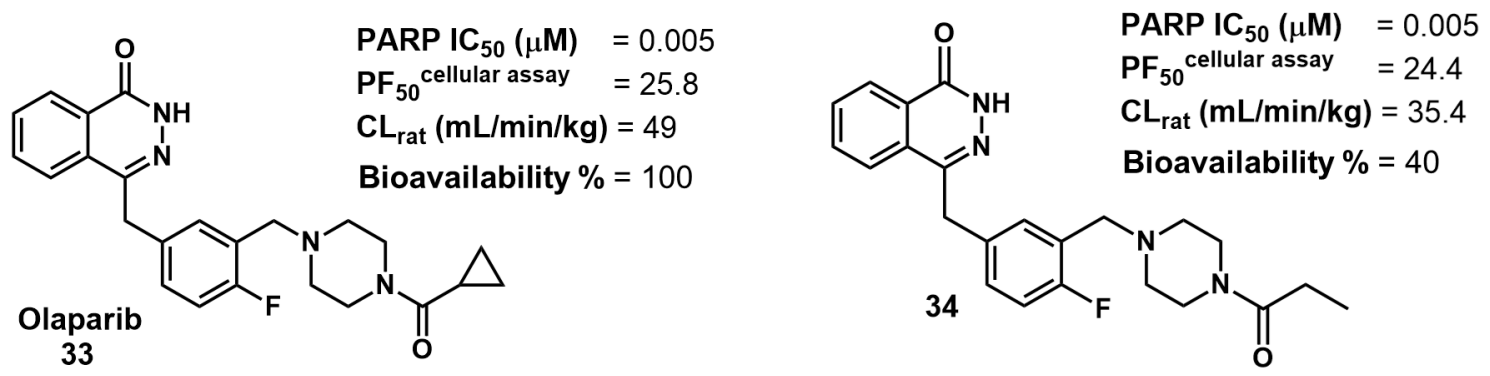

Figure 4 - PARP inhibitors. (a) PARP cocrystal structure for niraparib (PDB 4R6E) overlapping the PARP cocrystal structure for a nicotinamide related inhibitor (2-methylquinazolin-4(3H)-one) (PDB 4GV7) showing essential hydrogen bonds between inhibitors and the residues SER904 and GLY863. (b) PK and biological parameters for representative compounds generated during niraparib development. (c) PK and biological parameters for selected compounds generated during olaparib development. Abbreviations - PARP: poly (ADP-ribose) polymerase, $\mathrm{IC}_{50}$ : half maximal inhibitory concentration, $\mathrm{CL}_{\text {rat }}: \mathrm{clearance}$ in rats, $\mathrm{CL}_{\text {int }}$ : in vitro intrinsic clearance, $\mathrm{PF}_{50}$ : potentiation factor (calculated as the ratio of the $\mathrm{IC}_{50}$ growth curve for the alkylating agent methyl methane-sulfonate (MMS) divided by the $\mathrm{IC}_{50}$ of the curve of MMS + PARP inhibitor), $\mathrm{EC}_{50}$ : half maximal effective concentration. 
in the cellular assay (Fig. 4b) (Jones et al. 2015). Both compounds presented cytotoxicity against BCRA-silenced HeLa cells, yielding a selectivity index of 10 over non-silenced cell lines.

Pharmacokinetics in vitro parameters diverged significantly between compounds $\mathbf{2 4}$ and $\mathbf{2 5}$ (clearance on rat liver microsomes of $177 \mu \mathrm{L} /$ $\mathrm{min} / \mathrm{mg}$ for compound 24 and $28 \mu \mathrm{L} / \mathrm{min} / \mathrm{mg}$ for compound 25) (Jones et al. 2015). Nevertheless, both compounds showed high clearance on in vivo rat models $(450 \mathrm{~mL} / \mathrm{min} / \mathrm{kg}$ for compound 24 and $220 \mathrm{~mL} / \mathrm{min} / \mathrm{kg}$ for compound 25), which raised the hypothesis of extrahepatic metabolism. To investigate this assumption, clearance studies were performed on lung, kidney, intestine and heart microsomes, which revealed a turnover through the lungs and kidneys. Additional metabolism studies showed that CYPs $1 \mathrm{~A} 1$ and $1 \mathrm{~A} 2$ were responsible for oxidation reactions on compounds 24 and 25 (Jones et al. 2015).

A new batch of compounds was designed with the view of blocking the sites of oxidation (Jones et al. 2015). Appending a 3-substituted piperidine at the para position of the phenyl group (Fig. 4b) was the best strategy, which led to compound 26, $\left(\mathrm{IC}_{50}\right.$ enzimatic assay $^{2}=3.2 \mathrm{nM} ; \mathrm{EC}_{50}{ }^{\text {PARylation }}=24 \mathrm{nM}$; rat liver microsome clearance $=11 \mu \mathrm{L} / \mathrm{min} / \mathrm{mg}$; and rat clearance $=47 \mathrm{~mL} / \mathrm{min} / \mathrm{kg}$ ). The enantiomeric separation of compound $\mathbf{2 6}$ led to the identification of niraparib (the $S$ enantiomer of compound 26), which presented adequate in vitro metabolism, activity against the enzyme, improved cytotoxicity and inhibition of PARylation (Fig. 4b) (Jones et al. 2015). Based on the suitable preclinical findings, compound 26 (niraparib) entered clinical trials in 2008 and was granted FDA approval in 2017.

Olaparib (compound 33), a PARP inhibitor approved in 2014, was also developed by the simultaneous optimization of PD and PK properties (multiparametric optimization). Similar to the niraparib case, an SBDD approach relying on nicotinamide was used in the early phases of the discovery of olaparib. By constraining the rotation of the amide group, a phthalazinone (compound 27, Fig. 4c) having moderate activity against PARP $\left(\mathrm{IC}_{50}=0.77 \mu \mathrm{M}\right)$ was identified (Loh et al. 2005). This inhibitor had no activity in cellular assays, and therefore, new analogues were proposed using molecular modeling methods (compounds 2830). This strategy yielded compound 31 (Loh et al. 2005), a potent PARP inhibitor $\left(\mathrm{IC}_{50}\right.$ enzymatic assay $^{2}$ $0.007 \mu \mathrm{M}$ and potentiation factor, $\left.\mathrm{PF}_{50}=12.6\right)$. In parallel, the pharmacokinetics of the compounds was optimized to reduce the high in vitro metabolic rates of earlier analogues, such as compound $\mathbf{3 2}$ (Menear et al. 2008). The gathered results allowed the homopiperazine $\mathbf{3 1}$ to be evaluated in clinical trials in combination with cytotoxic agents via intravenous administration (Fig. 4c).

Next, new analogues were designed to obtain compounds suitable for oral administration (Dang et al. 2017). Approximately 1000 compounds were designed with the aim to improve essential physicochemical properties for gastrointestinal absorption. Therefore, molecular weight and polar surface area cutoffs were set to $550 \mathrm{Da}$ and 140 $\mathrm{A}^{2}$, respectively. Rotatable bonds and hydrogen bond donor/acceptors were limited to 7 and 10, respectively. Lipophilicity and $\mathrm{p} K_{\mathrm{a}}$ were balanced to achieve aqueous solubility below $0.1 \mathrm{mg} / \mathrm{mL}$.

Molecular modeling studies on the designed series indicated that the distal homopiperazine nitrogen could be functionalized to reduce its $\mathrm{p} K_{\mathrm{a}}$ $\left(\mathrm{p} K_{\mathrm{a}}=9.87\right)$, which could improve gastrointestinal absorption. Piperazines containing a cyclopropyl ring (compound 33) or an ethyl group (compound 34) proved to be the compounds with the best balance between enzyme inhibition, activity against whole cells, and in vivo plasma levels after oral administration (Fig. 4c). The PK profile was established for these compounds, and compound 33 displayed the highest oral exposure. Compound 33 (olaparib) was characterized using in vitro cellular assays, showing an $\mathrm{IC}_{50}$ value of $5 \mathrm{nM}$ 
(PARP inhibition) and $\mathrm{PF}_{50}$ of 25.8. Furthermore, compound $\mathbf{3 3}$ had a more pronounced cytotoxic effect on MDA-MB-468 BRCA-deficient cells in comparison with MDA-MB-231 BRCA-proficient cell lines (Menear et al. 2008). Compound 33 was effective towards in vivo xenograft models of cancer and, therefore, was selected for clinical development as a promising therapy for BRCAdeficient cancer (Menear et al. 2008). In 2014, olaparib was granted FDA approval for advanced BRCA-mutated ovarian cancer.

\section{Perspectives on drugging challenging targets}

Some proteins are categorized as challenging targets because of specific features that prevent them from being modulated by drug-like compounds. These features are the lack of deep and ordered pockets and the localization in intracellular compartments that are not accessible for potential ligands (Dang et al. 2017). Proteins that perform their biological activities by interacting with other proteins through protein-protein interactions (PPIs) are examples of molecular targets that lack well-behaved binding pockets (Scott et al. 2016). Therefore, modulating PPIs is a task that imposes different challenges when compared with traditional molecular targets. The approval of venetoclax (compound 35, Fig. 5a) in 2016 (Souers et al. 2013) as a modulator of the $\mathrm{Bcl}-2$ protein family has proven that is possible to overcome the challenges presented by these molecular targets.

Because of the presence of large PPI interfaces, RAS proteins are another example of actively pursued molecular targets that fall in this category of challenging targets. RAS proteins are GTPases involved in cellular signaling. Several biochemical pathways are RAS-dependent, and some of these pathways are important in cancer genesis, such as the mitogen-activated protein kinase (MAPK) cascade. This intracellular signaling route regulates cell growth and division. Another important system is the PI3K-AKT-mTOR pathway, which controls cell cycle and apoptosis and has direct implications in malignant transformation (Cox et al. 2014). The intense efforts in targeting these pathways follow the robust evidence that link RAS mutations and cancer. RAS mutations are found in $30 \%$ of human cancers and in $90 \%$ of tumors having a poor prognostic signature, such as pancreatic ductal adenocarcinomas (Cox et al. 2014).

No RAS inhibitor has reached the clinic despite three decades of intense research. Rigosertib is currently in phase III clinical trials for myelodysplastic syndrome (MDS), chronic myelomonocytic leukemia, and other conditions, and thus has the potential to be the first approved RAS inhibitor (Clinical studies for rigosertib 2017).

The RAS binding domain (RBD) PPI inhibitor rigosertib is a derivative of styryl benzyl sulfone and was identified in target-agnostic phenotypic screenings using a tumor cell cytotoxicity assay (Reddy et al. 2011). The hit compound (36) was selected on the basis of its ability to target cancer cells and its minor toxicity to normal cells (Fig. 5b). Molecular optimization was conducted by evaluating a library of 2000 (E)-styryl benzyl sulfones against cell lines from human prostate (DU145) and leukemic (K562) cancers. SAR derived from these results showed that nature, number and position of substituents in the two aromatic rings of the parent molecule were the key factors affecting cytotoxicity (Reddy et al. 2011). Compound 37 (rigosertib), the best candidate (Fig. 5b), presented $\mathrm{CC}_{50}$ values ranging from $10 \mathrm{nM}$ to $150 \mathrm{nM}$ on 58 cancer cell lines and no toxicity on normal cells. Moreover, this molecule presented suitable in vivo $\mathrm{PK}$ and PD parameters and a safety profile that endorsed its progression to clinical trials (Reddy et al. 2011).

While rigosertib proceeded to clinical trials, studies on its mechanism of action were performed (Chapman et al. 2012). Some reports demonstrated that this compound inhibits the PI3K-AKT pathway 


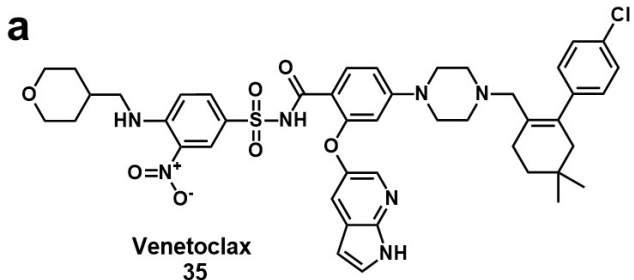

35

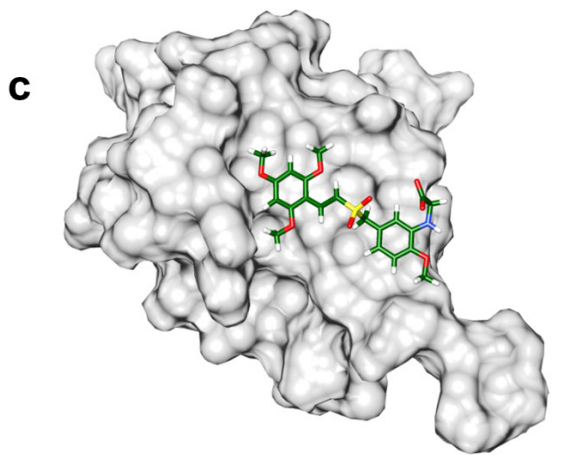

b

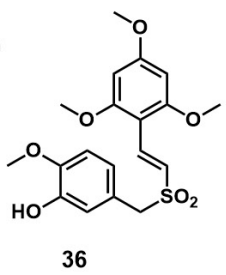

36

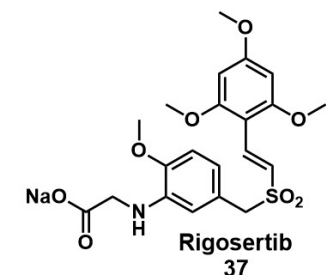

58 tumor cell lines $\mathrm{CC}_{50}(\mu \mathrm{M})-0.01-0.15$ Fibroblast $\quad \mathrm{CC}_{50}(\mu \mathrm{M})>100$

Xenograft tumor (BT20) growth inhibition $=80 \%$

Figure 5 - Drugging challenging targets. (a) Structure of venetoclax, the first PPI inhibitor targeting Bcl-2 proteins. (b) Rigosertib and its parent compound. (c) NMR structure for rigosertib in complex with the with RAS Binding Domain RBD (PDB 5J18). (d) PROTAC_ERR $\alpha$ structure; the parent VHL ligand is denoted by a blue dotted box, and the parent ERR $\alpha$ ligand is denoted by a pink dotted box. The structure of the E3 ubiquitin ligase VHL is depicted in blue (PDB 4WQO), and the structure of the estrogen-related receptor $\alpha$ ERR $\alpha$ is depicted in pink (PDB 3K6P). Abbreviations $-\mathrm{CC}_{50}$ : half maximal cytotoxic concentration.

(Chapman et al. 2012). Recently, rigosertib was identified as a RAS-mimetic that binds to the RBD region of RAS effectors, thus inhibiting RASdependent pathways (Athuluri-Divakar et al. 2016). A recently solved nuclear magnetic resonance (NMR) structure of the B-RAF kinase RBD in complex with rigosertib (Fig. 5c) showed the ligand occupying the interaction surface between RAF and RAS (Athuluri-Divakar et al. 2016). These data provide valuable structural information that can be used to design novel ligands that inhibit RAS-dependent downstream pathways.

Another promising approach to handle challenging molecular targets is the so-called small molecule proteolysis targeting chimeras (PROTACs). These agents are bi-functional molecules that induce the ubiquitination of proteins to signalize them for proteasome degradation (Gadd et al. 2017). Recent advances have been made in designing PROTACs with drug-like properties.
This strategy features several advantages, including the ability of targeting undruggable proteins, overcoming resistance mechanisms related to overexpression of inhibited proteins, and the potential of reducing systemic drug exposure (Lai and Crews 2016). One of the several small-molecule PROTACs that are under development has shown promising activity in mouse models of cancer (compound 38, Fig. 5d). The parent compound was identified using in silico and fragment-based screenings and binds to VHL E3, a ubiquitin ligase that induces the degradation of the estrogen-related receptor- $\alpha(E R R \alpha)(B o n d e s o n$ et al. 2015). ERR $\alpha$ is an orphan receptor that regulates cellular energy metabolism and that is related to the emergence of breast cancer (Stein et al. 2008). Based on the recent reported advances in PROTAC-based research, this field represents a novel and promising frontier to be explored in cancer drug discovery. 


\section{CONCLUSION}

Intense research on the improvement of the existing treatments for cancer have been currently conducted. Recent clinical trials and IND applications have shown that targeted therapy is the main focus of current cancer R\&D, in detriment of cytotoxic therapy. Comparing the anticancer drugs approved in the past five years with all the approved treatments demonstrates a sharp emergence of targeted therapies. A fraction of $45 \%$ of all the mAbs available in the market has been approved in this timeline, and $94 \%$ of new drugs are classified as targeted therapies. Although this type of drug has the advantage of having better selectivity over cytotoxic compounds, targeted therapies still lack satisfactory effectiveness. Therefore, considering the drawbacks and virtues of targeted and cytotoxic agents, the use of combination therapies incorporating both types of drugs has proved to be a worthwhile approach. Another important emerging strategy is the use of antibody-drug conjugates. This approach has been explored as an effective way to improve selectivity for cancer cells and consequently reduce the adverse effects associated with the use of cytotoxic agents.

Current drug discovery has brought to the market innovative drugs that exert their pharmacological effects through unprecedented mechanisms of action. This is illustrated by several examples: (i) the discovery of rigosertib, a breakthrough RAS inhibitor currently undergoing phase III clinical trials; (ii) the development of PARP inhibitors following key discoveries on the role of these proteins in BRCA-mutated tumors; (iii) and the approval of venetoclax, the first PPI inhibitor targeting Bcl-2 proteins. Despite the advances that have been made in the field of challenging targets, represented by the development of venetoclax, most of these proteins remain poorly explored. Limitations associated with their 3D structure along with their cellular localization consist of the main factors that render the development of effective drug-like compounds a remarkably complex task. Along with the progress that has been made in understanding cancer molecular biology, novel tools such as the PROTACs have emerged as a promising alternative to handle these difficult molecular targets.

The data examined herein show that the development of new anticancer drugs faces outstanding discoveries, with several agents featuring unique mechanisms of action reaching the market and progressing to clinical trials. As a result, the therapeutic arsenal for cancer has improved alongside with the robustness of the ongoing R\&D programs. These efforts might deliver in the upcoming years better solutions to those who suffer from this devastating disease.

\section{REFERENCES}

AGGARWAL S. 2010. Targeted cancer therapies. Nat Rev Drug Discov 9: 427-428.

AMES BN, SHIGENAGA MK AND HAGEN TM. 1993. Oxidants, antioxidants, and the degenerative diseases of aging. P Natl Acad Sci 90: 7915-7922.

ASGHAR U, WITKIEWICZ AK, TURNER NC AND KNUDSEN ES. 2015. The history and future of targeting cyclin-dependent kinases in cancer therapy. Nat Rev Drug Discov 14: 130-146.

ATHULURI-DIVAKAR SK ET AL. 2016. A Small Molecule RAS-Mimetic Disrupts RAS Association with Effector Proteins to Block Signaling. Cell 165: 643-655.

BACH PB. 2009. Limits on Medicare's ability to control rising spending on cancer drugs. N Engl J Med 360: 626-633.

BAGULEY B AND KERR D. 2001. Anticancer drug development, 1st ed., Cambridge: Academic Press, 397 p.

BARVIAN M ET AL. 2000. Pyrido[2,3-d]pyrimidin-7-one inhibitors of cyclin-dependent kinases. J Med Chem 43: 4606-4616.

BECK A, GOETSCH L, DUMONTET C AND CORVAÏA N. 2017. Strategies and challenges for the next generation of therapeutic antibodies. Nat Rev Immunol 16: 315-337.

BHINDER B AND DJABALLAH H. 2014. Drug discovery and repurposing at memorial sloan kettering cancer center: Chemical biology drives translational medicine. ACS Chem Biol 9: 1394-1397.

BONDESON DP ET AL. 2015. Catalytic in vivo protein knockdown by small-molecule PROTACs. Nat Chem Biol 11: 611-617. 
CHAPMAN CM ET AL. 2012. ON 01910.Na is selectively cytotoxic for chronic lymphocytic leukemia cells through a dual mechanism of action involving PI3K/AKT inhibition and induction of oxidative stress. Clin Cancer Res 18: 1979-1991.

CHARI RVJ. 2007. Targeted Cancer Therapy: Conferring Specificity to Cytotoxic Drugs. Accounts Chem Res 41: 98-107.

CHARI RVJ, MARTELL BA, GROSS JL, COOK SB, SHAH SA, BLÄTTLER WA, MCKENZIE SJ AND GOLDMACHER VS. 1992. Immunoconjugates Containing Novel Maytansinoids: Promising Anticancer Drugs. Cancer Res 52: 127-131.

CHEN P ET AL. 2016. Spectrum and Degree of CDK Drug Interactions Predicts Clinical Performance. Mol Cancer Ther 15: 2273-2281.

CLINICAL STUDIES FOR RIGOSERTIB. 2017. Available at: $<$ https://clinicaltrials.gov/ct2/results?term=Rigosertib $>$. Last accessed 22 Sep 2017.

COLOMBO M AND PERETTO I. 2008. Chemistry strategies in early drug discovery: an overview of recent trends. Drug Discov Today 13: 677-684.

COX AD, FESIK SW, KIMMELMAN AC, LUO J AND DER CJ. 2014. Drugging the undruggable RAS: Mission Possible? Nat Rev Drug Discov 13: 828-851.

DANG CV, REDDY PE, SHOKAT KM AND SOUCEK L. 2017. Drugging the "undruggable" cancer targets. Nat Rev Cancer 17: 502-508.

DEININGER M, BUCHDUNGER E AND DRUKER BJ. 2005. Review in translational hematology: The development of imatinib as a therapeutic agent for chronic myeloid leukemia. Blood 105: 2640-2653.

DIMASI JA, GRABOWSKI HG AND HANSEN RW. 2016. Innovation in the pharmaceutical industry: New estimates of R\&D costs. J Health Econ 47: 20-33.

DUMONTET C AND JORDAN MA. 2010. Microtubulebinding agents: a dynamic field of cancer therapeutics. Nat Rev Drug Discov 9: 790-803.

FINN RS ET AL. 2009. PD 0332991, a selective cyclin D kinase 4/6 inhibitor, preferentially inhibits proliferation of luminal estrogen receptor-positive human breast cancer cell lines in vitro. Breast Cancer Res 11: R77.

FISHKIN N, MALONEY EK, CHARI RVJ AND SINGH R. 2011. A novel pathway for maytansinoid release from thioether linked antibody-drug conjugates (ADCs) under oxidative conditions. Chem Commun 47: 10752-10754.

FOJO T, MAILANKODY S AND LO A. 2014. Unintended consequences of expensive cancer therapeutics: The pursuit of marginal indications and a me-too mentality that stifles innovation and creativity. JAMA Otolaryngol 140: 1225-1236.

GADD MS, TESTA A, LUCAS X, CHAN KH, CHEN W, LAMONT DJ, ZENGERLE M AND CIULLI A. 2017.
Structural basis of PROTAC cooperative recognition for selective protein degradation. Nat Chem Biol 13: 514-521.

GOODMAN J AND WALSH V. 2001. The story of taxol: Nature and politics in the pursuit of an anti-cancer drug, 1st ed., Cambridge: Cambridge University Press, $282 \mathrm{p}$.

JONES P ET AL. 2009. Discovery of 2-\{4-[(3S)-piperidin3-yl]phenyl $\}-2 \mathrm{H}$-indazole-7-carboxamide (MK-4827): A novel oral poly(ADP-ribose)polymerase (PARP) inhibitor efficacious in BRCA-1 and -2 mutant tumors. J Med Chem 52: 7170-7185.

JONES P, WILCOXEN K, ROWLEY M AND TONIATTI C. 2015. Niraparib: A Poly(ADP-ribose) polymerase (PARP) inhibitor for the treatment of tumors with defective homologous recombination. J Med Chem 58: 3302-3314.

JOO WD, VISINTIN I AND MOR G. 2013. Targeted cancer therapy: Are the days of systemic chemotherapy numbered? Maturitas 76: 308-314.

JORDAN A, HADFIELD JA, LAWRENCE NJ AND MCGROWN AT. 1998. Anticancer drugs: Agents which interact with the mitotic spindle. Med Res Rev 18: 259296.

KINCH MS. 2014. An analysis of FDA-approved drugs for oncology. Drug Discov Today 19: 1831-1835.

LAI AC AND CREWS CM. 2016. Induced protein degradation: An emerging drug discovery paradigm. Nat Rev Drug Discov 16: 101-114.

LAMBERT JM AND CHARI RVJ. 2014. Ado-trastuzumab emtansine (T-DM1): An antibody-drug conjugate (ADC) for HER2-positive breast cancer. J Med Chem 57: 69496964.

LEWIS PHILLIPS GD ET AL. 2008. Targeting HER2-positive breast cancer with trastuzumab-DM1, an antibodycytotoxic drug conjugate. Cancer Res 68: 9280-9290.

LOH VM ET AL. 2005. Phthalazinones. Part 1: The design and synthesis of a novel series of potent inhibitors of poly(ADP-ribose)polymerase. Bioorg Med Chem Lett 15: 2235-2238.

LU H AND SCHULZE-GAHMEN U. 2006. Toward understanding the structural basis of cyclin-dependent kinase 6 specific inhibition. J Med Chem 49: 3826-3831.

MAGALHAES LG, MARQUES FB, FONSECA MB, ROGÉRIO KR, GRAEBIN CS AND ANDRICOPULO AD. 2016. Discovery of a Series of Acridinones as Mechanism-Based Tubulin Assembly Inhibitors with Anticancer Activity. PLoS ONE 11: e0160842.

MASUI K, GINI B, WYKOSKY J, ZANCA C, MISCHEL PS, FURNARI FB AND CAVENEE WK. 2013. A tale of two approaches: Complementary mechanisms of cytotoxic and targeted therapy resistance may inform next-generation cancer treatments. Carcinogenesis 34: 725-738.

MENEAR KA ET AL. 2008. Novel Bioavailable Inhibitor of Poly (ADP-ribose) Polymerase-1. J Med Chem 51: 65816591. 
MERZ KM, RINGE D AND REYNOLDS C. 2010. Drug design: Structure- and ligand-based approaches, 1st ed., Cambridge: Cambridge University Press, 286 p.

MILLER DR. 2006. A tribute to Sidney Farber: The father of modern chemotherapy. Brit J Haematol 134: 20-26.

MOFFAT JG, RUDOLPH J AND BAILEY D. 2014. Phenotypic screening in cancer drug discovery: Past, present and future. Nat Rev Drug Discov 13: 588-602.

MURAI J, HUANG SN, DAS BB, RENAUD A, ZHANG Y, DOROSHOW JH, JI J, TAKEDA S AND POMMIER Y. 2012. Trapping of PARP1 and PARP2 by clinical PARP inhibitors. Cancer Res 72: 5588-5599.

NAHTA R AND ESTEVA FJ. 2003. HER-2-targeted therapy: Lessons learned and future directions. Clin Cancer Res 9: 5078-5084.

OLDHAM RK AND DILLMAN RO. 2008. Monoclonal antibodies in cancer therapy: 25 Years of progress. J Clin Oncol 26: 1774-1777.

PETRELLI A AND GIORDANO S. 2008. From single- to multi-target drugs in cancer therapy: When aspecificity becomes an advantage. Curr Med Chem 15: 422-432.

PRASAD V, DE JESUS K AND MAILANKODY S. 2017. The high price of anticancer drugs: Origins, implications, barriers, solutions. Nat Rev Clin Oncol 14: 381-390.

RAJU TN. 2000. The nobel chronicles. Lancet 355: 1022.

REDANA S, DONADIO M, NOLÈ F, JACOMUZZI ME, BEANO A, MARTINELLO R, SPINO A, VIALE G, AGLietTA M AND MONTEMURRO F. 2010. Trastuzumab with either docetaxel or vinorelbine as firstline treatment for patients with HER2-positive advanced breast cancer: A retrospective comparison. BMC Cancer 10: 28 .

REDDY MVR, VENKATAPURAM P, MALLIREDDIGARI MR, PALLELA VR, COSENZA SC, ROBELL KA, AKULA B, HOFFMAN BS AND REDDY EP. 2011. Discovery of a clinical stage multi-kinase inhibitor sodium (E)-2-\{2-methoxy-5-[(2',4',6'-trimethoxystyrylsulfonyl) methyl] phenylamino acetate (ON 01910.Na): Synthesis, structure-activity relationship, and biological activity. J Med Chem 54: 6254-6276.

RICHMOND A AND SU Y. 2008. Mouse xenograft models vs GEM models for human cancer therapeutics. Dis Model Mech 1: 78-82.

SANCAR A, LINDSEL-BOLTZ LA, ÜNSAL-KAÇMAZ K AND LINN S. 2004. Molecular mechanisms of mammalian
DNA repair and the DNA damage checkpoints. Annu Rev Biochem 73: 39-85.

SCOTT DE, BAYLY AR, ABELL C AND SKIDMORE J. 2016. Small molecules, big targets: Drug discovery faces the protein-protein interaction challenge. Nat Rev Drug Discov 15: 533-550.

SOUERS A ET AL. 2013. ABT-199, a potent and selective BCL-2 inhibitor, achieves antitumor activity while sparing platelets. Nat Med 19: 202-208.

STEIN RA, CHANG C, KAZMIN DA, WAY J, SCHROEDER T, WERGIN M, DEWHIRST MW AND MCDONNELL DP. 2008. Estrogen-related receptor $\alpha$ is critical for the growth of estrogen receptor-negative breast cancer. Cancer Res 68: 8805-8812.

SULLIVAN R AND AGGARWAL A. 2016. Health policy: Putting a price on cancer. Nat Rev Clin Oncol 13: 137-138.

TALMADGE JE, SINGH RK, FIDLER IJ AND RAZ A. 2007. Murine models to evaluate novel and conventional therapeutic strategies for cancer. Am J Pathol 170: 793804.

TONIATTI C, JONES P, GRAHAM H, PAGLIARA B AND GRAETTA G. 2014. Oncology drug discovery: Planning a turnaround. Cancer Discov 4: 397-404.

TOOGOOD PL ET AL. 2005. Discovery of a potent and selective inhibitor of cyclin-dependent kinase 4/6. J Med Chem 48: 2388-2406.

TOZER GM, KANTHOU C AND BAGULEY BC. 2005. Disrupting tumour blood vessels. Nat Rev Cancer 5: 423435.

TRIPATHY D, BARDIA A AND SELLERS WR. 2017. Ribociclib (LEE011): Mechanism of action and clinical impact of this selective cyclin-dependent kinase 4/6 inhibitor in various solid tumors. Clin Cancer Res 23: 3251-3262.

VENKITARAMAN AR. 2002. Cancer susceptibility and the functions of BRCA1 and BRCA2. Cell 108: 171-182.

WAHLBERG E ET AL. 2012. Family-wide chemical profiling and structural analysis of PARP and tankyrase inhibitors. Nat Biotechnol 30: 283-288.

WORLD HEALTH STATISTICS 2017. 2017. Available at: $<$ http://www.who.int/gho/publications/world health statistics/2017/en/>. Last accessed 20 Sep 2017.

WORLD POPULATION PROSPECTS THE 2015 REVISION. 2015. Available at: $<$ https://esa.un.org/unpd/ wpp/publications/files/key_findings_wpp_2015.pdf>. Last accessed 20 Sep 2017. 